\title{
Secondary attack rate of COVID-19 in household contacts in the Winnipeg Health Region, Canada
}

\author{
Krista Wilkinson $^{1}$ (D) $\cdot$ Xuan Chen $^{1} \cdot$ Souradet Shaw ${ }^{1}$ \\ Received: 22 July 2020 / Accepted: 3 November 2020 / Published online: 17 November 2020 \\ (C) The Canadian Public Health Association 2020
}

\begin{abstract}
Objectives Understanding important transmission parameters, such as secondary attack rates, can help in strategizing, prioritizing, and planning interventions to curb morbidity and mortality due to COVID-19. Early reports suggest the risk of transmission of SARS-CoV-2 (the pathogen that causes COVID-19 disease) is high among household contacts of cases. The purpose of this analysis was to determine the household transmissibility of SARS-CoV-2 in residents of the Winnipeg Health Region.

Methods We extracted information on COVID-19 cases and their household contacts from the regional public health surveillance database and used this dataset to calculate secondary attack rates.

Results We included 381 individuals from 102 unique households (102 primary cases and 279 household contacts). A total of 41 contacts from 25 households became secondary cases in the 14 days since last unprotected exposure to the primary case, resulting in a secondary attack rate of $14.7 \%$ (95\% CI: 10.5-18.8).

Conclusion Household transmission of SARS-CoV-2 has been an important contributor to the local COVID-19 epidemic.
\end{abstract}

\section{Résumé}

Objectifs Comprendre les importants paramètres de transmission, comme les taux d'infection secondaire, peut aider à établir des stratégies et des priorités et à planifier des interventions afin de réduire la morbidité et la mortalité liées à la COVID-19. Les premiers rapports laissent entendre que le risque de transmission du SRAS-CoV-2 (l'agent pathogène qui cause la COVID-19) est élevé parmi les contacts familiaux de cas de COVID-19. Le but de cette analyse visait à déterminer la transmissibilité familiale du SRAS-CoV-2 chez les résidents de la région sanitaire de Winnipeg.

Méthodes Nous avons tiré l'information sur les cas de COVID-19 et de leurs contacts familiaux de la base de données de surveillance de la santé publique à l'échelle régionale et nous avons utilisé ces données pour calculer les taux d'infection secondaire.

Résultats Nous avons inclus 381 personnes de 102 ménages uniques (102 cas primaires et 279 contacts familiaux). Au total, 41 contacts de 25 ménages sont devenus des cas secondaires dans les 14 jours depuis la dernière exposition non protégée au cas primaire, ce qui correspond à un taux d'infection secondaire de $14,7 \%$ (IC de $95 \%: 10,5-18,8$ ).

Conclusion La transmission familiale du SRAS-CoV-2 a contribué grandement à l'épidémie locale de la COVID-19.

Keywords COVID-19 $\cdot$ SARS-CoV-2 $\cdot$ Secondary attack rates $\cdot$ Household transmission $\cdot$ Emerging infectious diseases

Mots-clés COVID-19 · SRAS-CoV-2 $\cdot$ taux d'infection secondaire $\cdot$ transmission familiale $\cdot$ maladies infectieuses émergentes

\section{Introduction}

Krista Wilkinson

wilkinsk@myumanitoba.ca

1 Winnipeg Regional Health Authority, 490 Hargrave Street, Winnipeg, Manitoba R3A 0X7, Canada
Isolation of cases and contact tracing are critical in controlling the spread of infectious diseases such as COVID-19 in the community (Hellewell et al. 2020). Early reports suggest the risk of transmission of SARS-CoV-2 (the pathogen that causes COVID-19 disease) is high among household contacts of cases (Jing et al. 2020; COVID-19 National Emergency Response Center 2020). 
Winnipeg is the largest urban centre in the central Canadian province of Manitoba, and the Winnipeg Regional Health Authority (WRHA) provides healthcare for its 770,000 residents. The first case of COVID-19 in the Winnipeg Health Region (WHR) was identified in mid-March 2020 and by April 28, there had been 205 cases, for cumulative incidence of 26.6 (95\% CI: $23.1-30.5$ ) per 100,000 population, or an incidence density of 0.55 (95\% CI: $0.48-0.64$ ) per 100,000 person-days during this time period (i.e., 48 days).

Although the initial cases were largely associated with travel, cases were observed shortly after among their household contacts. The purpose of this analysis was to determine the proportion of COVID-19 cases among eligible household contacts, or secondary attack rate (SAR) of SARS-CoV-2, in residents of the WHR.

\section{Methods}

Laboratory testing for SARS-CoV-2 began in Manitoba in mid-January 2020 and was initially restricted to symptomatic individuals with a history of travel to Wuhan, China. By late March, testing had expanded to all symptomatic individuals with a history of travel outside Manitoba. Along with international travelers, testing at this time also included specific groups of symptomatic individuals lacking a travel exposure (e.g., healthcare workers, hospitalized in-patients). Testing of household contacts was limited to symptomatic individuals during the study period.

Manitoba Health, Seniors and Active Living (MHSAL) is the provincial ministry responsible for healthcare in Manitoba and receives reports for all cases of COVID-19 disease. Cases were defined as probable or confirmed as per the MHSAL COVID-19 protocol (Government of Manitoba 2020); a probable case met clinical and exposure criteria with an inconclusive laboratory report or met clinical criteria with an epi-link to a confirmed case or outbreak. A confirmed case had laboratory confirmation, with detection of at least one specific gene target by a NAAT assay. WHR cases were identified based on residential address and regional public health nurses conducted all case and contact interviews. Data entered into the regional electronic database included demographics, laboratory results, exposures, and contacts. Data were extracted on April 28, 2020.

In Manitoba, a close contact was defined as a person who provided care for, had close physical contact with, or had close prolonged contact to a probable or confirmed case while the case was ill (Government of Manitoba 2020). Close contacts were classified by type during the public health contact investigation; "Household contacts" were those identified as sharing a residence with a case.

Households were defined as a group of individuals named in a case's COVID-19 episode as household contacts. As contacts and cases may have been named across multiple episodes, we further linked groups by household individuals (i.e., if contact A was a named household contact to case B and also to case $\mathrm{C}$, we considered cases $\mathrm{B}$ and $\mathrm{C}$ as part of the same household).

The primary case was the case in the household with the earliest symptom onset. We assumed a minimum and maximum incubation period of 1 and 14 days respectively based on provincial guidelines (Government of Manitoba 2020); secondary cases were household contacts of the primary case with symptom onset $>1$ day after the primary case and up to 14 days after the primary case's symptom onset date. Any household members with symptoms developing one day or less after the primary case were defined as co-primary cases and were excluded from contact calculations. Contacts were followed for the 14-day period following the contact's last unprotected exposure to the primary case (i.e., from when the case became aware of COVID-19 status and began selfisolation). Households with primary cases occurring after April 14, 2020 were excluded as their contacts did not have a full incubation period to develop symptoms.

We calculated the SAR as the total number of secondary cases over the total number of eligible household contacts.

\section{Results}

We identified 400 individuals from 107 unique households. We classified 14 individuals who developed symptoms within one day of the primary case as co-primary cases and excluded them from further calculations. We excluded a further 5 primary cases with a household size of 1 (i.e., no household contacts). After exclusions, we included 381 individuals from 102 unique households: 102 primary cases and 279 household contacts.

There were no primary cases in the less than 10 years old age group (Table 1). There were two households with primary cases in the 10-19-year-old age group; one household had 2 adult contacts that remained asymptomatic and the other had four adult contacts of which 2 became cases. Adults were the primary cases in 100 (98\%) included households and transmitted to a single child in two unique households.

Of the primary cases, $43(42 \%)$ had a travel exposure, 27 (26\%) were contacts to known cases, $11(11 \%)$ had both a travel exposure and contact to a known case, 20 (20\%) had an unknown source of acquisition, and $1(1 \%)$ did not have an exposure history recorded.

There was a median of 3 household contacts per primary case (minimum 0, maximum 25) (Table 2). Figure 1 shows the variation in household size. The largest household (centre) was an outlier with 25 contacts of which 2 became secondary cases; members of this household ranged in age from 0 to 61 (mean age 28). The highest number of household contacts to become 
Table 1 Characteristics of COVID-19 cases and household contacts

\begin{tabular}{llll}
\hline & $\begin{array}{l}\text { Primary } \\
\text { case }(n=102)\end{array}$ & $\begin{array}{l}\text { Secondary } \\
\text { case }(n=41)\end{array}$ & $\begin{array}{l}\text { Asymptomatic } \\
\text { contacts }(n=238)\end{array}$ \\
\hline $\begin{array}{l}\text { Mean age (min/max) } \\
\text { Age group in years (\%) }\end{array}$ & $44(18 / 89)$ & $40(1 / 72)$ & $34(0 / 99)$ \\
$0-9$ & $0(0)$ & $2(5)$ & $25(11)$ \\
$10-19$ & $2(2)$ & $0(0)$ & $40(17)$ \\
$20-29$ & $26(26)$ & $14(35)$ & $51(22)$ \\
$30-39$ & $19(19)$ & $6(15)$ & $30(13)$ \\
$40-49$ & $18(18)$ & $5(12)$ & $21(9)$ \\
$50-59$ & $18(18)$ & $7(17)$ & $33(14)$ \\
$60-69$ & $11(11)$ & $5(12)$ & $18(8)$ \\
$70+$ & $7(7)$ & $1(3)$ & $18(8)$ \\
Male $(\%)$ & $41(40)$ & $22(54)$ & $88(37)$ \\
\hline
\end{tabular}

secondary cases was 4 , representing a SAR of $67 \%$ (4 of 6 household contacts became secondary cases); members of this household ranged in age from 5 to 43 (mean age 24).

The overall median time to symptom onset in secondary cases was 6 days; however, when looking at the first symptomatic individual in the household following the primary case, the median time to symptom onset was 5 days.

Primary and secondary cases were older than asymptomatic household contacts (Table 1); 4 (4\%) of the COVID-19 cases were under the age of 20 as compared to 65 (28\%) of the asymptomatic household members. A higher proportion of secondary cases were males compared to primary cases or asymptomatic household contacts.

A total of $74(72 \%)$ households had no observed household transmission. Forty-one household contacts from 25 unique households became secondary cases and 238 household contacts remained asymptomatic in the 14 days since last unprotected exposure to the case. The maximum number of contacts within a single household to convert to cases was 4 . The SAR in household contacts was $14.7 \%$ (95\% CI: 10.5-18.8).

\section{Discussion}

In this preliminary analysis of 102 unique households in the Winnipeg Health Region with COVID-19 cases, we report a SAR of $14.7 \%$ (95\% CI: $10.5-18.8)$.
Our results align with a study of 219 primary cases from Guangzhou, China, that reported a household attack rate of $13.8 \%$ (11.1-17.0) for close relatives and 19.3\% (15.5-23.9) if the contact lived at the same residential address (Jing et al. 2020). Another report from Shenzhen, China, reported a SAR of $11.2 \%(9.1-13.8)$ among household contacts (Bi et al. 2020); in this analysis, they assumed that contacts missing a test result were not infected, which was analogous to our approach in which we assume an asymptomatic/non-tested contact was not a case.

In the WHR, almost all primary cases were adults (98\%), which aligned with a study of family clusters in Greece (91\%) (Maltezou et al. 2020). We observed the prevalent direction of transmission to be from adult to child, which may be partially attributed to the low number of households with children as primary cases. Classes were suspended in Manitoba K-12 schools on March 23, 2020, which may have contributed to the low number of children as primary cases. There is emerging evidence that asymptomatic infection and mild clinical illness are more common in children than in adults (Du et al. 2020); this lower likelihood of symptomatic infection may also under-estimate SAR. Our analyses were restricted to laboratory-confirmed cases of COVID-19 and testing was restricted to symptomatic contacts of cases in Manitoba. We observed that $98 \%$ of the household contacts who were children remained asymptomatic and thus were not eligible for testing. If all household members (including asymptomatic
Table 2 Characteristics of included households $(n=102)$

\begin{tabular}{llll}
\hline & $\begin{array}{l}\text { Number of } \\
\text { households }\end{array}$ & Median & $\begin{array}{l}\text { Minimum/ } \\
\text { maximum }\end{array}$ \\
\hline Contacts per household & 102 & 3 & $1 / 25$ \\
Symptomatic contacts per household & 102 & 0 & $0 / 4$ \\
Days to symptom onset—all symptomatic contacts & 41 & 6 & $2 / 14$ \\
Days to symptom onset—first symptomatic contact & 25 & 5 & $2 / 14$ \\
\hline
\end{tabular}


Fig. 1 Social network diagram of households with COVID-19 cases in the Winnipeg Health Region; green dots represent primary cases, red dots represent secondary cases, and yellow dots represent asymptomatic household contacts; lines connect case(s) to named household contacts

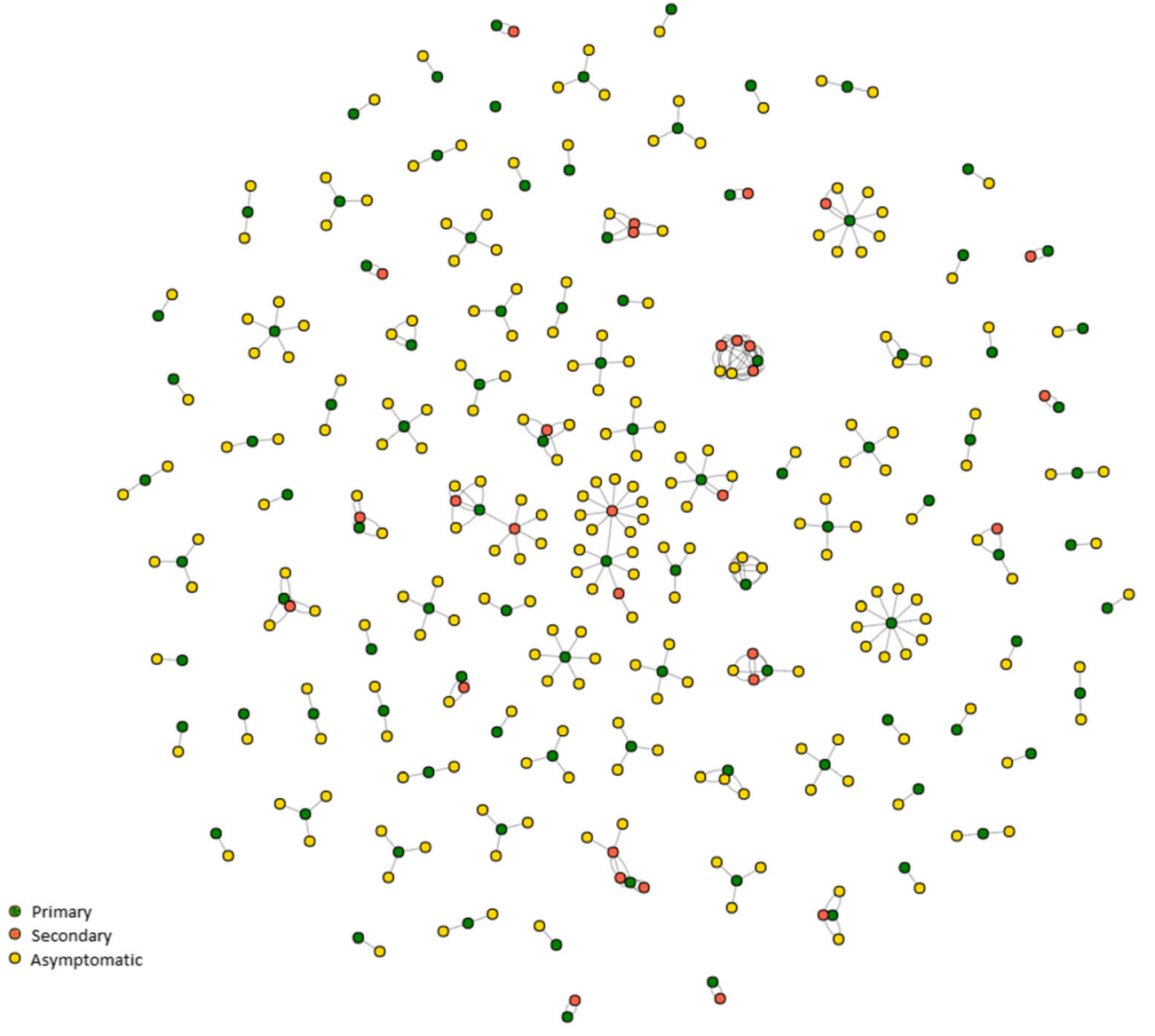

contacts) had been eligible for testing, the secondary attack rate might have been greater.

In Manitoba, self-isolation for 14 days following symptom onset and after last unprotected exposure was required for both cases and contacts respectively and alternative isolation accommodations were also provided for individuals not able to maintain isolation within their residences. These practices may have resulted in lower SAR estimates as the exposure risk to household contacts may have been minimized. The median number of total contacts (both household and non-household) per case in the WHR has consistently been three, and close household contacts have been the most common contact type reported, indicating that physical distancing has been effective in the province. The self-isolation of contacts also reduced the possibility of contacts in the household being exposed outside of the home, making it more likely that the primary case was the source of exposure.

Our study had some identified limitations. We classified any household member with symptom onset occurring more than one day after the index case as a secondary case; this may have resulted in an over-estimation of the SAR if secondary cases with the same exposures as primary cases had longer incubation periods. Of the 41 secondary cases we observed, $11(27 \%)$ had symptom onset within three days of the primary case; excluding those cases resulted in a SAR of $11.2 \%(95 \%$
CI: 7.4-15.0), which was lower but consistent with our point estimate.

We relied on classification of household contact as recorded in our electronic database and did not validate against residential address. We identified one large household with 25 contacts; it is possible that these individuals were not all residing at the same address or that there was movement of members between households and contacts may have had variable exposures to the primary case. Excluding this outlier from the analysis did not change the SAR (15.3\% [95\% CI: 10.9-19.8]).

\section{Conclusion}

Our report shows that household transmission of SARS-CoV2 has been an important contributor to the regional COVID-19 epidemic. In the WHR, the source of exposure moved quickly from the outset when most cases were attributed to travel, to the source of exposure being attributed to contact to a known case. Understanding important parameters, such as secondary attack rates and direction of transmission, can help align the local public health response with transmission dynamics with the goal of minimizing the morbidity and mortality due to COVID-19. 


\section{Compliance with ethical standards}

Conflict of interest The authors declare that they have no conflict of interest.

\section{References}

Bi, Q., Wu, Y., Mei, S., Ye, C., Zou, X., Zhang, Z., et al. (2020). Epidemiology and transmission of COVID-19 in 391 cases and 1286 of their close contacts in Shenzhen, China: a retrospective cohort study. The Lancet Infectious Diseases, 20(8), 911-919.

COVID-19 National Emergency Response Center, Epidemiology and Case Management Team, Korea Centers for Disease Control and Prevention (2020). Coronavirus Disease-19: Summary of 2,370 Contact Investigations of the First 30 Cases in the Republic of Korea. Osong Public Health and Research Perspectives, 11(2), 81-84. https://doi.org/10.24171/j.phrp.2020.11.2.04

Du, W., Yu, J., Wang, H., Zhang, X., Zhang, S., Li, Q., \& Zhang, Z. (2020). Clinical characteristics of COVID-19 in children compared with adults in Shandong Province, China. Infection, 48(3), 445-452. https://doi.org/10.1007/s15010-020-01427-2.
Government of Manitoba. (2020). Interim Guidance Public health measures: managing novel coronavirus (COVID-19) cases and contacts in community. https://manitoba.ca/asset_library/en/coronavirus/ interim_guidance.pdf. Accessed 23 Apr 2020.

Hellewell, J., Abbott, S., Gimma, A., Bosse, N. I., Jarvis, C. I., Russell, T. W., et al. (2020). Feasibility of controlling COVID-19 outbreaks by isolation of cases and contacts. The Lancet Global Health, 8(4), E488-496.

Jing, Q. L., Liu, M. J., Zhang, Z. B., Fang, L. Q., Yuan, J., Zhang, A. R., et al. (2020). Household secondary attack rate of COVID-19 and associated determinants in Guangzhou, China: a retrospective cohort study. The Lancet Infectious Diseases, 20(10), 1141-1150.

Maltezou, H. C., Vorou, R., Papadima, K., Kossyvakis, A., Spanakis, N., Gioula, G., Exindari, M., Metallidis, S., Lourida, A. N., Raftopoulos, V., Froukala, E., Martinez-Gonzalez, B., Mitsianis, A., Roilides, E., Mentis, A., Tsakris, A., \& Papa, A. (2020). Transmission dynamics of SARS-CoV-2 within families with children in Greece: a study of 23 clusters. Journal of Medical Virology. https://doi.org/10.1002/jmv.26394 Advance online publication.

Publisher's note Springer Nature remains neutral with regard to jurisdictional claims in published maps and institutional affiliations. 